\title{
Message from the Editor-in-Chief and Deputy Editors
}

\author{
Kunio Doi · Fujio Araki · Masahiro Endo • \\ Shigehiko Katsuragawa · Hideo Murayama $\cdot$ \\ Shigeru Sanada
}

Published online: 1 December 2007

(C) Japanese Society of Radiological Technology and Japan Society of Medical Physics 2007

Radiological Physics and Technology is the official English-language journal of the Japanese Society of Radiological Technology and the Japan Society of Medical Physics, which have a combined membership of more than 18,000 . Although a large fraction of the articles in this new journal may be written by members of these societies, we welcome contributions from authors in many countries around the world.

We believe that new ideas and new findings are the most important ingredients in scientific and technical publications. It is worthwhile to report new ideas and new findings as soon as possible, even if the supporting data might not be completely available at an early phase of research and development. Therefore, we welcome short articles clearly describing new ideas and new findings that are likely to have a significant impact on radiological physics and technology in the future. We are willing to take a small risk against a potentially large benefit to the societies. Authors can then publish long articles later, with comprehensive analysis and extensive data, which will provide strong evidence and support for their early findings.

We are strongly against unethical conduct of research, which includes fabrication of data and plagiarism in

K. Doi $(\bowtie)$

Department of Radiology, The University of Chicago,

Chicago, IL, USA

e-mail: k-doi@uchicago.edu

F. Araki · S. Katsuragawa

Kumamoto University, Kumamoto, Japan

M. Endo $\cdot$ H. Murayama

National Institute of Radiological Sciences, Chiba, Japan

S. Sanada

Kanazawa University, Kanazawa, Japan scientific publications. Manuscripts submitted for publication of original work should not include duplicates or copies of statements, graphs, and/or data from earlier work without proper reference to earlier articles, which may have been published in any language by the same or different authors. All of the editors of the 15 journals in the international organization of medical physics (IOMP), chaired by William Hendee, Ph.D., have basically agreed to take a strong stand against plagiarism. It is our intention to publish as an editorial in this journal the policy against plagiarism, once the IOMP decides to adopt such a policy.

The manuscripts accepted for publication in Radiological Physics and Technology are available through online first, which provides accepted articles in electronic form via the Internet prior to the appearance of the printed journal. Each article will have a permanent, unique international identification code, the digital object identification (DOI), which can be cited by the journal name and the DOI before the printed version becomes available. The printed version includes the DOI and the date of online publication.

Kunio Doi, Ph.D., Editor-in-Chief

Fujio Araki, Ph.D., Deputy Editor

Masahiro Endo, Ph.D., Deputy Editor

Shigehiko Katsuragawa, Ph.D., Deputy Editor

Hideo Murayama, Ph.D., Deputy Editor

Shigeru Sanada, Ph.D., DMsc, Deputy Editor 